FSU-HEP-930924-R

Revised January 1994

\title{
PRODUCTION OF CP-ODD HIGGS BOSONS WITH LARGE TRANSVERSE MOMENTUM AT HADRON SUPERCOLLIDERS
}

\author{
Chung Kaof \\ Department of Physics, B-159, Florida State University \\ Tallahassee, FL 32306-3016, USA
}

\begin{abstract}
A two Higgs doublet model is employed to study the production of a CP-odd Higgs boson $(A)$ associated with a large transverse momentum jet $(j)$ at hadron supercolliders. The cross section of $p p \rightarrow j A+X$ is evaluated with four subprocesses: $g g \rightarrow g A, g q \rightarrow q A, g \bar{q} \rightarrow \bar{q} A$ and $q \bar{q} \rightarrow g A$. We find that $p p \rightarrow j A+X$ is a significant source of CP-odd Higgs bosons at future hadron supercolliders.
\end{abstract}

*Internet Address: Kao@Fsuhep.physics.fsu.edu 


\section{Introduction}

In the Standard Model (SM) of electroweak interactions, only one Higgs doublet is required to generate masses for the fermions as well as the gauge bosons. A single neutral CP-even Higgs boson $\left(H^{0}\right)$ appears after the spontaneous symmetry breaking. Various extensions of the SM have more Higgs multiplets and lead to additional physical spin-0 fields [1].

A general two Higgs doublet model [2] has doublets $\Phi_{1}$ and $\Phi_{2}$ with vacuum expectation values (VEV's) $v_{1}$ and $v_{2}$. If CP is invariant in the Higgs sector, there remain five physical Higgs bosons [1] after symmetry breaking: a pair of singly charged Higgs bosons $H^{ \pm}$, two neutral CP-even scalars $H$ (heavier) and $h$ (lighter), and a neutral CP-odd pseudoscalar $A$.

Two models with a discrete symmetry [3] have been considered for the Yukawa interactions among the Higgs bosons and fermions. In model I [4, 6], all fermions couple to $\Phi_{2}$, and the $A q \bar{q}$ interaction takes the form

$$
\mathcal{L}_{A q \bar{q}}=+i \frac{m_{u}}{v} \cot \beta \bar{u} \gamma_{5} u A-i \frac{m_{d}}{v} \cot \beta \bar{d} \gamma_{5} d A
$$

In model II [5, 6], which is required in the minimal supersymmetry model (MSSM)币, $\Phi_{1}$ couples to down-type quarks and charged leptons while $\Phi_{2}$ couples to up-type quarks and neutrinos, and the $A q \bar{q}$ interaction is

$$
\mathcal{L}_{A q \bar{q}}=+i \frac{m_{u}}{v} \cot \beta \bar{u} \gamma_{5} u A+i \frac{m_{d}}{v} \tan \beta \bar{d} \gamma_{5} d A
$$

\footnotetext{
${ }^{\dagger}$ Reviews of the MSSM can be found in [7]- $[10]$. Recent studies on the search for MSSM Higgs bosons at hadron supercolliders are to be found in 11-15].
} 
where $\tan \beta \equiv v_{2} / v_{1}, \sqrt{v_{1}^{2}+v_{2}^{2}}=v=2 M_{W} / g, u$ and $d$ are generic u-type and d-type quarks. Without loss of generality, Model II is chosen in all our calculations. In this model, the $A d \bar{d}$ coupling is enhanced when $\tan \beta$ is large. The cross section for Model I is usually dominated by the contribution from the top quark, and can be well approximated by our data at $\tan \beta=1$ multiplied by $\cot ^{2} \beta$.

At hadron supercolliders, such as the SSC in the USA and the LHC at CERN, the SM Higgs boson can be produced dominantly from gluon fusion [16], and from vector boson fusion [17]-[19]. The $A$ does not couple to the gauge bosons at the tree level, therefore, gluon fusion and heavy quark fusion [20] are the two major sources of the $A$ in high energy hadron collisions.

\section{CP-odd Higgs Bosons with Large $P_{T}$}

If the SM Higgs boson $\left(H^{0}\right)$ can be produced in association with a large transverse momentum jet $(j), p p \rightarrow j H^{0}+X$, via the following subprocesses: $g g \rightarrow g H^{0}, g q \rightarrow q H^{0}, g \bar{q} \rightarrow \bar{q} H^{0}$, and $q \bar{q} \rightarrow g H^{0}$, the mass of the Higgs boson might be reconstructed from its $\tau^{+} \tau^{-}$decay channel 21] if the Higgs mass is in the intermediate range; or from the $Z Z$ decay mode 22 if the Higgs boson is heavier.

The same subprocesses can also produce the CP-odd Higgs bosons with large transverse momentum $\left(P_{T}\right)$ via triangle and box diagrams, as shown in Figure 1. The amplitudes of the quark loops can be expressed in terms

\footnotetext{
$\ddagger$ I would like to thank Uli Baur for comparing the matrix elements in this reference.
} 
of form factors [23] which are combinations of scalar one-loop integrals [24]. We have calculated all loop integrations with the computer code LOOP [25], which evaluates one loop integrals analytically and generates numerical data.

If there exist just three generations of quarks, only the top quark loop ( $t$-loop) and the bottom quark loop (b-loop) make significant contributions. Therefore, only the third generation quark loops are considered in our calculations with the following values of parameters: $\alpha=1 / 128, M_{Z}=91.17$ $\mathrm{GeV}, M_{W}=80.0 \mathrm{GeV}, \sin ^{2} \theta_{W}=0.230$, the bottom quark mass $m_{b}=5$ $\mathrm{GeV}$. If not specified, the top quark mass $\left(m_{t}\right)$ is taken to be $150 \mathrm{GeV}$. The mass of the CP-odd Higgs boson $\left(m_{A}\right)$ is considered to be between 50 and $1000 \mathrm{GeV}$. The updated parton distribution functions of Owens [26] with $\Lambda=0.177 \mathrm{GeV}$ and $Q^{2}=m_{A}^{2}+P_{T}^{2}$ are chosen to evaluate the cross section of $p p \rightarrow j A+X$ at the energies of the $\mathrm{SSC}(\sqrt{s}=40 \mathrm{TeV})$ and the LHC $(\sqrt{s}=14 \mathrm{TeV})$. Since it is at the order of $\alpha_{s}^{3}$, the cross section of $p p \rightarrow j A+X$ is very sensitive to the choice of $\Lambda$ and $Q^{2}$. To evaluate the production rate of $A$ with large $P_{T}$ as well as to avoid the singularities at $P_{T} \rightarrow 0$, we impose a $P_{T}$ cut on the $A$ and the jet: $P_{T}>50 \mathrm{GeV}$.

Figure 2 shows the cross section of $p p \rightarrow j A+X$ at the SSC and the LHC energies, as a function of $m_{A}$, for $m_{t}=150 \mathrm{GeV}$ and various values of $\tan \beta$. The cross section is about 5-19 times larger at $\sqrt{s}=40 \mathrm{TeV}$ than at $\sqrt{s}=14$ $\mathrm{TeV}$, for $50 \mathrm{GeV}<m_{A}<1000 \mathrm{GeV}$. The loop integrals are functions of the mass of the CP-odd Higgs boson $\left(m_{A}\right)$, the quark mass in the loops $\left(m_{q}\right)$, 
and the Mandelstam variables: $\hat{s}, \hat{t}, \hat{u}$. For $m_{A}<m_{t}$, the $t$-loop is almost independent of $m_{t}$, thus the amplitude is very sensitive to $\tan \beta$. For $\tan \beta$ larger than about 5, the cross section is dominated by the b-loop. At the threshold of $m_{A}=2 m_{t}$, the imaginary part of the amplitude is turned on. Therefore, the amplitude squared $\left(|M|^{2}\right)$ grows rapidly when $m_{A}$ is close to $2 m_{t}$. When $m_{q}^{2} \ll m_{A}^{2}<\hat{s}$, the amplitude squared behaves as

$$
\left|M_{q-l o o p}\right|^{2} \sim m_{q}^{4} \ln ^{4}\left(m_{q}^{2}\right)
$$

The $t$-loop dominates in a large region of $\tan \beta$. The cross section is almost proportional to $\cot ^{2} \beta$ for $\tan \beta<10$. Only for $\tan \beta$ close to $m_{t} / m_{b}$, can the $b$-loop dominate and the total cross section be enhanced by large $\tan \beta$. Not shown is the interference between the $t$-loop and the $b$-loop. The $t$-loop and the $b$-loop interfere destructively if $m_{A}$ is close to $2 m_{t}$, but constructively if $m_{A}$ is away from $2 m_{t}$.

To compare the production rate of the CP-odd Higgs boson $(A)$ to that of the SM Higgs boson $\left(H^{0}\right)$ at the SSC energy, we present the cross section of $p p \rightarrow \phi+X, \phi=H^{0}$ or $A$ from various subprocesses in Table I, for $m_{t}=150$ $\mathrm{GeV}$ and $\tan \beta=1$. Several interesting aspects can be learned from Table I and Figure 2: (1) If the Higgs bosons are produced from $g g \rightarrow \phi$, or the subprocesses of $p p \rightarrow j \phi+X$ via quark loops, the cross section of $A$ is at least twice that of the $H^{0}$ for $m_{\phi}<500 \mathrm{GeV}$. At $m_{\phi}=2 m_{t}$, the cross section of $A$ is about 5 times that of the $H^{0}$; which implies that the threshold enhancement at $2 m_{t}$ is much larger for the $A$ than for the $H^{0}$. For larger $m_{\phi}$, their cross 
sections are about the same. (2) $g g \rightarrow g \phi$ dominates and contributes about $80 \%$ to the cross section of $p p \rightarrow j \phi+X$. (3) For $m_{\phi}>400 \mathrm{GeV}$, the number of Higgs bosons produced from $g g \rightarrow g \phi+X$ with $P_{T}(\phi)>50 \mathrm{GeV}$, is almost comparable to that from $g g \rightarrow \phi$. (4) For $m_{\phi}>50 \mathrm{GeV}$, the cross sections of the $A$ and $H^{0}$ are the same from $g g \rightarrow \phi b \bar{b}$, which is a good approximation to the 'exact' cross section of $\phi$ produced from $b \bar{b}$ fusion [20]. (5) For $\tan \beta>10$, $g g \rightarrow A b \bar{b}$ becomes the major source of large $P_{T}$ CP-odd Higgs bosons. Its cross section is proportional to $\tan ^{2} \beta$ and it is greatly enhanced by large $\tan \beta$.

The effects of $\tan \beta$ and $m_{t}$ on the cross section of $p p \rightarrow j A+X$ at $\sqrt{s}=40 \mathrm{TeV}$ are shown in Figure 3, for $m_{t}=120,150$ and $180 \mathrm{GeV}$. Two values of $m_{A}$ are considered: (a) $m_{A}=200 \mathrm{GeV}$, which is less than $2 m_{t}$; and (b) $m_{A}=400 \mathrm{GeV}$, which is larger than $2 m_{t}$. If $\tan \beta$ is less than about 10, $m_{t}$ slightly affects the total cross section: larger $m_{t}$ slightly enhances the cross section for $m_{A}=200 \mathrm{GeV}$, but slightly reduces the cross section for $m_{A}=400 \mathrm{GeV}$. The total cross section is almost independent of $m_{t}$ for $\tan \beta>10$.

The top quark mass dependence on the matrix element squared of $g g \rightarrow$ $g H^{0}$ has been studied in detail [21, 22]. A similar study for $g g \rightarrow g A$ is currently under investigation. At the threshold of $2 m_{t}$, the enhancement on the $|M|^{2}$ for $g g \rightarrow g \phi$ is very similar to that of $g g \rightarrow \phi$, where $\phi=H^{0}$ or $A$. Therefore, we review and discuss the production of $p p \rightarrow \phi+X$ from gluon 
fusion in the appendix.

In Figure 4, we show the $P_{T}$ distribution of $p p \rightarrow g A+X$ from $g g \rightarrow g A$ at $\sqrt{s}=40 \mathrm{GeV}$ for $m_{t}=150 \mathrm{GeV}, m_{A}=200$ and $400 \mathrm{GeV}$. The effect of $\tan \beta$ is similar to that on the cross section versus $m_{A}$ in Fig. 2. If we require $P_{T}(A)>100 \mathrm{GeV}$, the cross section will be reduced to only about $1 / 3$ of that with $P_{T}>50 \mathrm{GeV}$ for $m_{A}<200 \mathrm{GeV}$ while about $1 / 2$ of $m_{A}>400$ GeV will survive.

\section{Large Quark Mass Limit ${ }^{\S}$}

If the quark mass in the loop diagrams is much larger than that of the CPodd Higgs boson, $m_{q} \gg m_{A}$, the $g g A$ and $g g g A$ couplings can be obtained from the low energy theorem of the axial anomaly [27]-29] or from the exact calculation of $g g \rightarrow A$ at the limit of $m_{q}^{2} / m_{A}^{2} \gg 1$. An effective Lagrangian [30, 31] can be written as

$$
\mathcal{L}_{e f f}=\frac{\lambda}{8} G_{\mu \nu}^{a} \tilde{G}^{a \mu \nu} A
$$

where $\lambda=\frac{\alpha_{s}}{2 \pi v}$ and $\tilde{G}^{a \mu \nu}=\epsilon^{\mu \nu \rho \sigma} G_{\rho \sigma}^{a}$.

Applying the effective Lagrangian, we obtain the amplitude squared for various subprocesses at the large quark mass limit

$$
\begin{aligned}
|M(g g \rightarrow g A)|^{2} & =\frac{3 f}{32}\left(\frac{s^{4}+t^{4}+u^{4}+m_{A}^{8}}{s t u}\right) \\
|M(g q \rightarrow q A)|^{2} & =\frac{f}{24}\left[-\left(\frac{s^{2}+u^{2}}{t}\right)\right]
\end{aligned}
$$

\footnotetext{
$\S$ In this section, $\tan \beta$ is taken to be $1 ; \alpha_{s}$ is taken to be $\frac{12 \pi}{23} / \ln \left(Q^{2} / \Lambda^{2}\right)$; and only the top quark loop is considered.
} 


$$
|M(q \bar{q} \rightarrow g A)|^{2}=\frac{f}{9}\left(\frac{t^{2}+u^{2}}{s}\right)
$$

where $f=\lambda^{2} g_{s}^{2}$ and the $|M|^{2}$ has been summed and averaged over all spins and colors.

Table II shows the $P_{T}$ distribution $\left(d \sigma / d P_{T}\right)$ of $p p \rightarrow g A+X$, at $\sqrt{s}=14$ $\mathrm{TeV}$, from the top quark loops of $g g \rightarrow g A$, for various $m_{t}$ and $m_{A}$, with $\tan \beta=1$. The large quark mass limit is a very good approximation for $P_{T}<m_{t}$ and $m_{A}<m_{t}$. It provides a good check for the exact calculation. It slightly underestimates the exact cross section if $m_{A}$ is close to $2 m_{t}$ and $P_{T}$ is less than $2 m_{t}$, because the exact cross section is enhanced by the threshold effect. However, it overestimates the cross section if $P_{T}$ is larger than $2 m_{t}$ or if $m_{A}$ is larger than about $4 m_{t}$. Similar results have been found for the SM Higgs boson 21, 22].

\section{Conclusions}

The $p p \rightarrow j A+X$ is a very significant source of CP-odd Higgs bosons at future hadron colliders for $\tan \beta$ less than about 10, especially for the detection modes that require a large $P_{T}$ for the $A$. The large quark mass limit of $p p \rightarrow j A+X$ is a good approximation for $m_{A}<m_{t}$, but overestimates the exact cross section for large $m_{A}$ and $P_{T}$. In the region of small $P_{T}$, a complete description requires higher order corrections [31]-[33], and the resummation of gluon emission 34-36.

If the $A b \bar{b}$ coupling is proportional to $\tan \beta, g g \rightarrow A b \bar{b}$ is the dominant process to produce the $A$ for $\tan \beta>10$ and $m_{A}>100 \mathrm{GeV}$. The subpro- 
cesses $g g \rightarrow g A$ and $g g \rightarrow A b \bar{b}$ can be considered as complementary to each other for producing large $P_{T}$ CP-odd Higgs bosons at future hadron colliders.

The total cross section presented in this letter for $p p \rightarrow j A+X$ is less reliable for a much heavier $A$. It is likely to overestimate the production rates due to large $\ln \left(m_{A}^{2} / P_{T 0}^{2}\right)$ contributions, since a constant minimal transverse momentum cutoff $\left(P_{T}>P_{T 0}=50 \mathrm{GeV}\right)$ has been applied.

The total cross section of $p p \rightarrow j A+X$ at $\sqrt{s}=14 \mathrm{TeV}$ is about 5-19 times smaller than at $\sqrt{s}=40 \mathrm{TeV}$, for $50 \mathrm{GeV}<m_{A}<1000 \mathrm{GeV}$.

\section{Acknowledgements}

I am grateful to Howie Baer, Bill Bardeen, Uli Baur, Joe Polchinski and Xerxes Tata for beneficial discussions, to Sally Dawson and Duane Dicus for continuing encouragement as well as valuable comments and to Harvey Goldman for technical support. This research was supported in part by the DOE contract DE-FG05-87-ER40319. 


\section{Appendix}

At the lowest order, the cross sections of $g g \rightarrow \phi$ via the top quark loops are

$$
\begin{aligned}
\sigma\left(g g \rightarrow H^{0}\right) & =\frac{1}{64}\left(\frac{\alpha_{s}^{2} \alpha_{W}}{M_{W}^{2}}\right)(s)|F(\rho)|^{2} \delta\left(s-M_{H}^{0}{ }^{2}\right) \\
\sigma(g g \rightarrow A) & =\frac{1}{64}\left(\frac{\alpha_{s}^{2} \alpha_{W}}{M_{W}^{2}}\right)(s)|G(\rho)|^{2} \delta\left(s-M_{A}^{2}\right)
\end{aligned}
$$

where $\alpha_{W}=\alpha / \sin ^{2} \theta_{W}$ and $\rho=m^{2} / m_{\phi}^{2}$.

The functions $F(\rho)$ and $G(\rho)$ are

$$
\begin{aligned}
& F(\rho)=+\rho[2+(4 \rho-1) I(\rho)] \\
& G(\rho)=-\rho I(\rho)
\end{aligned}
$$

and the function $I(\rho)$ is

$$
\begin{aligned}
I(\rho)= & +\int_{0}^{1} \frac{d y}{y}\left\{\ln \left[1-\frac{y(1-y)}{\rho-i \epsilon}\right]\right\} \\
= & -2\left[\sin ^{-1}\left(\frac{1}{2 \sqrt{\rho}}\right)\right]^{2}, \rho \geq \frac{1}{4} \\
& +\frac{1}{2}\left[\ln \left(\frac{z_{+}}{z_{-}}\right)-i \pi\right]^{2}, \rho<\frac{1}{4}
\end{aligned}
$$

where $z_{ \pm}=[1 \pm \sqrt{1-4 \rho}] / 2$.

I. When $m$ is very small, $\rho \ll 1$, therefore,

$$
I(\rho)=\frac{1}{2}\left[\ln ^{2}\left(\frac{s}{m^{2}}\right)-\pi^{2}-2 i \pi \ln \left(\frac{s}{m^{2}}\right)\right] .
$$

\footnotetext{
I In this appendix, $m=m_{t} ; \tan \beta=1$; and $\phi$ is the SM Higgs boson $H^{0}$ or a CP odd Higgs boson $A$.
} 
II. At the threshold, $\rho=1 / 4$, therefore

$$
\begin{aligned}
I\left(\frac{1}{4}\right) & =-\pi^{2} / 2, \\
F\left(\frac{1}{4}\right) & =+\frac{1}{2} \\
G\left(\frac{1}{4}\right) & =+\frac{\pi^{2}}{8} .
\end{aligned}
$$

III. At very large $m, \rho \gg 1$, therefore,

$$
\begin{aligned}
I(\rho) & =-\frac{1}{2 \rho}-\frac{1}{24 \rho^{2}}+O\left(\frac{1}{\rho^{3}}\right), \\
F(\rho) & =+\frac{1}{3}+O\left(\frac{1}{\rho}\right), \\
G(\rho) & =+\frac{1}{2}+O\left(\frac{1}{\rho}\right) .
\end{aligned}
$$

Therefore, at the threshold of $m_{\phi}=2 m_{t}$,

$$
\frac{\sigma(g g \rightarrow A)}{\sigma\left(g g \rightarrow H^{0}\right)}=\pi^{4} / 16 \simeq 6
$$

while at the large top quark mass limit,

$$
\frac{\sigma(g g \rightarrow A)}{\sigma\left(g g \rightarrow H^{0}\right)}=9 / 4
$$

The same ratios appear in the cross sections of $g g \rightarrow g \phi$ at both limits. 


\section{References}

[1] G. Gunion, H. Haber, G. Kane and S. Dawson, The Higgs Hunter's Guide, Addison-Wesley, Redwood City, CA (1990).

[2] H. M. Georgi, Hadronic J. 1 (1978) 155.

[3] S. L. Glashow and S. Weinberg, Phys. Rev. D15 (1977) 1958.

[4] H. E. Haber, G. L. Kane and T. Stirling, Nucl. Phys. B161 (1979) 493.

[5] J. F. Donoghue and L. F. Li, Phys. Rev. D19 (1979) 945.

[6] L. Hall and M. Wise, Nucl. Phys. B187 (1981) 397.

[7] H. P. Nilles, Phys. Rep. 110 (1984) 1.

[8] P. Nath, R. Arnowitt and A. Chamseddine, Applied $N=1$ Supergravity, ICTP Series in Theoretical Physics, Vol. I, World Scientific (1984).

[9] H. Haber and G. Kane, Phys. Rep. 117 (1985) 75.

[10] X. Tata, in The Standard Model and Beyond, p. 304, edited by J. E. Kim, World Scientific (1991).

[11] V. Barger, M. Berger, A. Stange and R. Phillips, Phys. Rev. D45 (1992) 4128 . 
[12] J. Gunion, R. Bork, H. Haber and A. Seiden, Phys. Rev. D46 (1992) 2040; J. Gunion and L. Orr, Phys. Rev. D46 (1992) 2052; J. Gunion, H. Haber and C. Kao, Phys. Rev. D46 (1992) 2907.

[13] Z. Kunszt and F. Zwirner, Nucl. Phys. B385 (1992) 3.

[14] H. Baer, M. Bisset, C. Kao and X. Tata, Phys. Rev. D46 (1992) 1067; H. Baer, M. Bisset, D. Dicus, C. Kao and X. Tata, Phys. Rev. D47 (1993) 1062; H. Baer, C. Kao and X. Tata, Phys. Lett. B303 (1993) 284.

[15] J. Dai, J. F. Gunion and R. Vega, Phys. Lett. B315 (1993) 355.

[16] H. M. Georgi, S. L. Glashow, M. E. Machacek and D. V. Nanopoulos, Phys. Rev. Lett. 40 (1978) 692.

[17] R. Cahn and S. Dawson, Phys. Lett. B136 (1984) 196.

[18] M. Chanowitz and M. K. Gaillard, Phys. Lett. B142 (1984) 85; Nucl. Phys. B261 (1985) 379.

[19] G. L. Kane, W. Repko and W. Rolnick, Phys. Lett. B148 (1984) 367.

[20] D. A. Dicus and S. Willenbrock Phys. Rev. D39 (1989) 751.

[21] R. Ellis, I. Hinchliffe, M. Soldate and J. van der Bij, Nucl. Phys. B297 (1988) 221.

[22] U. Baur and E. W. N. Glover, Nucl. Phys. B339 (1990) 38. 
[23] G. Passarino and M. Veltman, Nucl. Phys. B160 (1979) 151.

[24] G. 't Hooft and M. Veltman, Nucl. Phys. B153 (1979) 365.

[25] D. Dicus and C. Kao, LOOP, a FORTRAN program for doing one-loop integrals with momenta in the numerator, unpublished, (1991).

[26] J. F. Owens, Phys. Lett. B266 (1991) 126.

[27] S. L. Adler, Phys. Rev. 177 (1969) 2426.

[28] J. Bell and R. Jackiw, Nuovo Cim. A60 (1969) 47.

[29] S. L. Adler and W. A. Bardeen, Phys. Rev. 182 (1969) 1517.

[30] A. Djouadi, M. Spira and P. M. Zerwas, DESY preprint, DESY-92-170.

[31] R. P. Kauffman and W. Schaffer, BNL preprint, BNL-49061.

[32] S. Dawson, Nucl. Phys. B359 (1991) 283.

[33] A. Djouadi, M. Spira and P. M. Zerwas, Phys. Lett. B264 (1991) 440; D. Graudenz, M. Spira and P. M. Zerwas, Phys. Rev. Lett. 70 (1993) 1372.

[34] I. Hinchliffe and S. F. Novaes, Phys. Rev. D38 (1988) 3475.

[35] R. P. Kauffman, Phys. Rev. D44 (1990) 1415; D45 (1992) 1512.

[36] C. P. Yuan, Phys. Lett. B283 (1992) 395. 


\section{Tables}

TABLE I. The cross section of $p p \rightarrow \phi+X$ at $\sqrt{s}=40 \mathrm{TeV}$, in $p b$, as a function of $m_{\phi}$, where $\phi$ is the SM Higgs boson $\left(H^{0}\right)$ or the CP-odd Higgs boson $(A)$. Various subprocesses are considered with $m_{t}=150 \mathrm{GeV}$ and $\tan \beta=1$. We have imposed a cut on $P_{T}(\phi)=P_{T}>50 \mathrm{GeV}$ for $p p \rightarrow j \phi+X$ and $p p \rightarrow \phi b \bar{b}+X$.

\begin{tabular}{lccccccc}
\hline$m_{\phi}(\mathrm{GeV})$ & 50 & 100 & 200 & 300 & 400 & 600 & 800 \\
\hline Raw Cross Section & & & & & & & \\
$g g \rightarrow H^{0}$ & 310 & 120 & 52 & 47 & 40 & 9.6 & 2.7 \\
$g g \rightarrow H^{0} t \bar{t}$ & 27 & 7.7 & 1.4 & 0.68 & 0.44 & 0.21 & 0.11 \\
$g g \rightarrow H^{0} b \bar{b}$ & 43 & 7.8 & 1.0 & 0.27 & 0.097 & 0.021 & $6.7 \times 10^{-3}$ \\
$P_{T}>50 \mathrm{GeV}$ & & & & & & & \\
$g g \rightarrow H^{0} b \bar{b}$ & 2.5 & 1.0 & 0.26 & 0.091 & 0.039 & 0.010 & $3.6 \times 10^{-3}$ \\
$g g \rightarrow g H^{0}$ & 64 & 40 & 23 & 22 & 21 & 6.2 & 2.0 \\
$g q \rightarrow q H^{0}$ & 10 & 6.5 & 3.7 & 3.6 & 3.3 & 1.0 & 0.33 \\
$g \bar{q} \rightarrow \bar{q} H^{0}$ & 4.4 & 2.7 & 1.4 & 1.3 & 1.1 & 0.31 & 0.092 \\
$q \bar{q} \rightarrow g H^{0}$ & 0.17 & 0.12 & 0.041 & $7.2 \times 10^{-3}$ & $1.9 \times 10^{-3}$ & $2.8 \times 10^{-4}$ & $6.7 \times 10^{-5}$ \\
\hline $\mathrm{Raw}$ Cross Section & & & & & & & \\
$g g \rightarrow A$ & 620 & 270 & 130 & 290 & 87 & 14 & 3.6 \\
$g g \rightarrow A t \bar{t}$ & 6.9 & 4.6 & 2.2 & 1.2 & 0.71 & 0.29 & 0.13 \\
$g g \rightarrow A b \bar{b}$ & 46 & 7.9 & 1.0 & 0.27 & 0.097 & 0.021 & $6.7 \times 10^{-3}$ \\
$P_{T}>50 \mathrm{GeV}$ & & & & & & & \\
$g g \rightarrow A b \bar{b}$ & 2.6 & 1.0 & 0.26 & 0.091 & 0.039 & 0.010 & $3.6 \times 10^{-3}$ \\
$g g \rightarrow g A$ & 136 & 89 & 58 & 125 & 46 & 9.4 & 2.7 \\
$g q \rightarrow q A$ & 22 & 15 & 9.6 & 21 & 7.5 & 1.6 & 0.45 \\
$g \bar{q} \rightarrow \bar{q} A$ & 9.5 & 6.1 & 3.7 & 7.6 & 2.6 & 0.47 & 0.12 \\
$q \bar{q} \rightarrow g A$ & 0.36 & 0.25 & 0.11 & 0.042 & $4.5 \times 10^{-3}$ & $5.1 \times 10^{-4}$ & $1.1 \times 10^{-4}$ \\
\hline
\end{tabular}


TABLE II. The $P_{T}$ distribution $\left(d \sigma / d P_{T}\right)$ of $p p \rightarrow g A+X$ in $\mathrm{pb} / \mathrm{GeV}$, at $\sqrt{s}=14 \mathrm{TeV}$, as a function of $m_{A}$ and $m_{t}$. We consider only the top quark loop diagrams of $g g \rightarrow g A$ with $\tan \beta=1$.

\begin{tabular}{lcccccc}
\hline$m_{t} \backslash P_{T}(\mathrm{GeV})$ & 100 & 200 & 400 & 600 & 800 & 1000 \\
\hline$m_{A}=100 \mathrm{GeV}$ & & & & & & \\
100 & $1.1 \times 10^{-1}$ & $8.9 \times 10^{-3}$ & $2.3 \times 10^{-4}$ & $1.5 \times 10^{-5}$ & $1.6 \times 10^{-6}$ & $2.4 \times 10^{-7}$ \\
150 & $1.0 \times 10^{-1}$ & $1.2 \times 10^{-2}$ & $4.0 \times 10^{-4}$ & $3.1 \times 10^{-5}$ & $3.8 \times 10^{-6}$ & $6.0 \times 10^{-7}$ \\
200 & $9.9 \times 10^{-2}$ & $1.3 \times 10^{-2}$ & $5.6 \times 10^{-4}$ & $4.9 \times 10^{-5}$ & $6.4 \times 10^{-6}$ & $1.1 \times 10^{-6}$ \\
$\infty$ & $9.5 \times 10^{-2}$ & $1.2 \times 10^{-2}$ & $8.1 \times 10^{-4}$ & $1.2 \times 10^{-4}$ & $2.5 \times 10^{-5}$ & $6.3 \times 10^{-6}$ \\
$m_{A}=400 \mathrm{GeV}$ & & & & & & \\
100 & $1.2 \times 10^{-2}$ & $2.2 \times 10^{-3}$ & $1.4 \times 10^{-4}$ & $1.4 \times 10^{-5}$ & $1.7 \times 10^{-6}$ & $2.7 \times 10^{-7}$ \\
150 & $3.3 \times 10^{-2}$ & $6.2 \times 10^{-3}$ & $4.4 \times 10^{-4}$ & $4.3 \times 10^{-5}$ & $5.6 \times 10^{-6}$ & $9.1 \times 10^{-7}$ \\
200 & $6.5 \times 10^{-2}$ & $1.2 \times 10^{-2}$ & $8.5 \times 10^{-4}$ & $8.8 \times 10^{-5}$ & $1.2 \times 10^{-5}$ & $2.0 \times 10^{-6}$ \\
$\infty$ & $1.2 \times 10^{-2}$ & $2.8 \times 10^{-3}$ & $3.8 \times 10^{-4}$ & $7.5 \times 10^{-5}$ & $1.8 \times 10^{-5}$ & $5.0 \times 10^{-6}$ \\
$m_{A}=600 \mathrm{GeV}$ & & & & & & \\
100 & $1.8 \times 10^{-3}$ & $3.9 \times 10^{-4}$ & $4.0 \times 10^{-5}$ & $5.4 \times 10^{-6}$ & $8.5 \times 10^{-7}$ & $1.5 \times 10^{-7}$ \\
150 & $5.0 \times 10^{-3}$ & $1.1 \times 10^{-3}$ & $1.3 \times 10^{-4}$ & $1.8 \times 10^{-5}$ & $2.9 \times 10^{-6}$ & $5.4 \times 10^{-7}$ \\
200 & $1.0 \times 10^{-2}$ & $2.3 \times 10^{-3}$ & $2.7 \times 10^{-4}$ & $3.9 \times 10^{-5}$ & $6.5 \times 10^{-6}$ & $1.2 \times 10^{-6}$ \\
$\infty$ & $4.7 \times 10^{-3}$ & $1.2 \times 10^{-3}$ & $2.0 \times 10^{-4}$ & $4.6 \times 10^{-5}$ & $1.3 \times 10^{-5}$ & $3.8 \times 10^{-6}$ \\
$m_{A}=1000 \mathrm{GeV}$ & & & & & & \\
100 & $1.1 \times 10^{-4}$ & $2.7 \times 10^{-5}$ & $4.0 \times 10^{-6}$ & $8.1 \times 10^{-7}$ & $1.8 \times 10^{-7}$ & $4.2 \times 10^{-8}$ \\
150 & $3.2 \times 10^{-4}$ & $8.2 \times 10^{-5}$ & $1.3 \times 10^{-5}$ & $2.7 \times 10^{-6}$ & $6.2 \times 10^{-7}$ & $1.5 \times 10^{-7}$ \\
200 & $6.9 \times 10^{-4}$ & $1.7 \times 10^{-4}$ & $2.9 \times 10^{-5}$ & $6.2 \times 10^{-6}$ & $1.5 \times 10^{-6}$ & $3.6 \times 10^{-7}$ \\
$\infty$ & $1.1 \times 10^{-3}$ & $3.0 \times 10^{-4}$ & $5.7 \times 10^{-5}$ & $1.6 \times 10^{-5}$ & $5.2 \times 10^{-6}$ & $1.8 \times 10^{-6}$ \\
\hline & & & & & &
\end{tabular}




\section{Figures}

FIG. 1 The Feynman diagrams of the subprocesses (a) $g g \rightarrow g A$, (b) $g q \rightarrow q A$ and (c) $q \bar{q} \rightarrow g A$. We have not shown the diagrams with various permutations of the external legs.

FIG. 2 The cross section of $p p \rightarrow j A+X$ in $p b$ versus $m_{A}$, for $m_{t}=150$ $\mathrm{GeV}, \tan \beta=1,2,5,10$ and 30 , at the energies: (a) $\sqrt{s}=40 \mathrm{TeV}$ and (b) $\sqrt{s}=14 \mathrm{TeV}$.

FIG. 3 The cross section of $p p \rightarrow j A+X$ in $p b$ versus $\tan \beta$, at $\sqrt{s}=40$ $\mathrm{TeV}$, for $m_{t}=120$ (dotted), 150(solid) and 180 (dashed) GeV. Two masses of the CP-odd Higgs boson are considered: (a) $m_{A}=200 \mathrm{GeV}$ and (b) $m_{A}=400 \mathrm{GeV}$.

FIG. 4 The $P_{T}$ distribution of $p p \rightarrow g A+X$ from $g g \rightarrow g A$ at $\sqrt{s}=40$ $\mathrm{TeV}$, for $m_{t}=150 \mathrm{GeV}$ and $\tan \beta=1,2,5,10$ and 30 . Two masses of the CP-odd Higgs boson are considered: (a) $m_{A}=200 \mathrm{GeV}$ and (b) $m_{A}=400$ GeV. 
This figure "fig1-1.png" is available in "png" format from: http://arxiv.org/ps/hep-ph/9310206v2 
This figure "fig1-2.png" is available in "png" format from: http://arxiv.org/ps/hep-ph/9310206v2 
This figure "fig1-3.png" is available in "png" format from: http://arxiv.org/ps/hep-ph/9310206v2 
This figure "fig1-4.png" is available in "png" format from: http://arxiv.org/ps/hep-ph/9310206v2 